\title{
Evaluation of spectrum of MRI findings in children with Hypoxic Ischemic Encephalopathy and its comparison with transcranial sonography
}

\author{
Mathur M. ${ }^{1}$, Gupta I. ${ }^{*}$, Mittal D. ${ }^{3}$ \\ DOI: https://doi.org/10.17511/ijmrr.2021.i02.06 \\ 1 Manoj Mathur, Professor, Department of Radiodiagnosis, Govt. Medical College and Rajindra Hospital, Patiala, Punjab, India. \\ 2* Ishita Gupta, Junior Resident, Department of Radiodiagnosis, Govt. Medical College and Rajindra Hospital, Patiala, Punjab, India. \\ 3 Dimple Mittal, Amravati Enclave, Near Choukhi Dhani, Panchkula, Haryana, India.
}

\begin{abstract}
Background: Hypoxic ischemic encephalopathy is a serious concern among asphyxiated newborns due to the associated long term sequelae like cognitive impairment and cerebral palsy. Though the incidence of hypoxic injury remains higher in preterm babies due to incomplete brain maturation, it can occur in term babies as well despite institutional deliveries due to an array of unavoidable fetal, maternal and placental causes. Aims: This study was conducted as an attempt to evaluate the risk factors, to study the correlation between the term of pregnancy with TCUS and MRI imaging findings in HIE and characterise patterns of CNS involvement. Materials and methods: It was a crosssectional study carried on 50 neonates with clinically diagnosed HIE presenting to the Department of Radiodiagnosis, Rajindra Hospital Patiala who were subjected to transcranial sonography and MRI. Results and Conclusion: This study demonstrated term infants have significant involvement of basal ganglia thalamus type (central) pattern of involvement and preterm infants have periventricular leukomalacia type (white matter injury) of a pattern of involvement. The overall sensitivity and specificity of TCUS in detecting imaging findings in children with clinically diagnosed HIE compared to MRI was found to be $70.45 \%$ and $50 \%$ respectively, yielding the overall diagnostic accuracy of TCUS as $68 \%$ compared to MRI. TCUS can depict central and white matter abnormalities better than peripheral lesions. However MRI provides additional diagnostic information in many cases and can detect precisely the extent of brain injury.
\end{abstract}

Keywords: Hypoxic Ischemic Encephalopathy, Periventricular Leukomalacia, Transcranial Ultrasound, Magnetic Resonance Imaging, Germinal Matrix Hemorrhage

\section{Corresponding Author}

Ishita Gupta, Junior Resident, Department of Radiodiagnosis, Govt. Medical College and Rajindra Hospital, Patiala, Punjab, India. Email: gishita8@gmail.com

\section{How to Cite this Article}

Mathur M, Gupta I, Mittal D. Evaluation of spectrum of MRI findings in children with Hypoxic Ischemic Encephalopathy and its comparison with transcranial sonography. Int J Med Res Rev. 2021;9(2):87-96. Available From

https://ijmrr.medresearch.in/index.php/ijmrr/article/ view/1274
To Browse

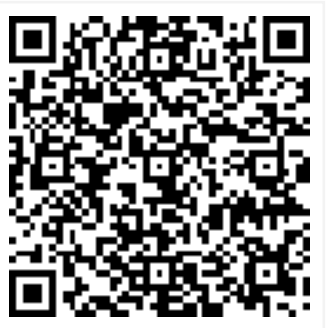

Manuscript Received 2021-03-28

Conflict of Interest No

(C) 2021 by Manoj Mathur Ishita
Review Round 1 2021-03-30

Funding

$\mathrm{Nil}$

Review Round 2
2021-04-10
Ethical Approval
Yes

Review Round 2

Yes
Review Round 3

Plagiarism X-checker $6 \%$
Accepted 2021-04-20

Note 


\section{Introduction}

Neonatal Hypoxic-Ischemic Encephalopathy designates the clinical and neuropathologic findings following either intrapartum or neonatal asphyxia [1].

As per the American Academy of Paediatrics and the American College of Obstetrics and Gynaecologists Hypoxic Ischemic Encephalopathy (HIE) is clinically diagnosed according to the following essential criteria:

- Profound metabolic or mixed acidemia

- 5 minute APGAR score of 0-3

- Neonatal neurological sequelae

- Multiorgan involvement [2]

Hypoxic-ischemic injury in full-term infants accounts for approximately $15 \%-20 \%$ of neonatal mortality, and $25 \%$ of those who survive to demonstrate significant neurological deficits. It occurs in 5\% of preterm infants. Up to $19 \%$ of infants born before 28 weeks of gestation develop cerebral palsy [3]

A widely used clinical classification of HIE devised by Sarnat and Sarnat (1976) classifies HIE into 3 stages.

- Stage I: characterised by hyperalertness, uninhibited Moro and stretch reflexes, sympathetic effects and normal Electroencephalogram (EEG).

- Stage II: infant has obtundation, hypotonia, strong distal flexion and multifocal seizures. The EEG shows a perirolandic pattern sometimes preceded by continuous delta activity.

- Stage III: the infant is stuporous and flaccid, with suppressed brainstem and autonomic functions. The EEG is iso-potential or has infrequent periodic discharges [4]

Clinically diagnosed infants with signs and symptoms of hypoxic-ischemic insult can be imaged by Transcranial Ultrasound (TCUS), Computerized Tomography and Magnetic Resonance Imaging (MRI) during the neonatal period. Transcranial ultrasound is cheap, easily available, portable, bedside modality, especially useful in acute setting in critically ill infants in neonatal intensive care units. It is used as the first screening modality, is ideal for follow up of the evolution of changes within the brain parenchyma and monitor the response to treatment.
MR due to its high spatial resolution, excellent inherent soft tissue contrast, multiplanar imaging capability and lack of ionising radiations is an excellent modality in imaging of asphyxiated neonates. It has a higher detection rate and can precisely estimate the location and extent of lesions in HIE [5].

Despite advances in medical infrastructure and increasing institutional deliveries in developing countries, hypoxic-ischemic injury remains a common problem and major cause of neonatal mortality and long term adverse neurological outcome. This study aimed to evaluate the spectrum of MRI findings and to determine the sensitivity of transcranial ultrasound in comparison to MRI in children with clinically diagnosed hypoxicischemic injury.

\section{Materials and Methods}

Study Population: This was a cross-sectional study carried out at Government Medical College and Rajindra Hospital, Patiala. The study population consisted of 50 paediatric patients with HIE who were referred from the Department of Paediatrics. All clinically diagnosed hemodynamically stable children without general contraindications to MRI were included in the study.

Data Collection: The children were subjected to transcranial sonography and MRI. Transcranial ultrasound was done with Philips EPIQ 5 G ultrasound machine using sector or curved array transducer. MR images were obtained by $1.5 \mathrm{~T}$ MRI machine (Siemens magnetom aera) using the following sequences: Axial T1 weighted images (T1WI); axial, sagittal and coronal T2 weighted images (T2WI), Axial T2 FLAIR (Fluid Attenuated Inversion Recovery), axial DWI (Diffusion-Weighted Images), SWI (Susceptibility Weighted Images) and T1 MPRAGE (Magnetized Prepared Rapid Gradient Echo).

Data Analysis: The data was described in terms of range; frequencies (number of cases) and relative frequencies (percentages) as appropriate. For comparing categorical data, Chi-square (X2) test was performed and the exact test was used when the expected frequency is less than 5. A probability value ( $p$-value) less than 0.05 was considered statistically significant. All statistical calculations were done by SPSS (Statistical Package for the Social Science) SPSS 21 version statistical program for Microsoft Windows. 
Ethical Considerations: Ethical clearance was received from Research and Ethical Committee, Government Medical College, Patiala. The study was approved by the Faculty of Medical Sciences, Baba Farid University of Health Sciences, Faridkot on 29.05.2019 (BFUHS/2k19p-TH/7273)

\section{Results}

The present study consisted of 50 paediatric patients with clinically diagnosed HIE who were referred to the department of Radiodiagnosis, Rajindra Hospital Patiala.

Age distribution: Ranged from 8 days -2 months. The maximum number of children were in the $<1$ month age group (58\%). The mean age of children in our study was 28.28 days.

Gender distribution: 21 (42\%) of total children were females and 29 (58\%) were males.

Gestational age: 32 (64\%) children born as term and $18(36 \%)$ born as preterm were included.

Mode of delivery: $31(62 \%)$ children were born by Normal vaginal delivery (NVD), 17 (34\%) by Lower Segment Caesarean Section (LSCS) and 2 (4\%) by Assisted vaginal delivery.

Birth weight: In this study, out of a total of 18 preterms, a weight of 6 (33.33\%) ranged between $1.5-2 \mathrm{~kg}$ and $12(66.67 \%)$ ranged between $2-2.5 \mathrm{~kg}$. Out of a total of 32 term-born children, the weight of $23(72 \%)$ ranged between $2.5-3.5 \mathrm{~kg}$ and that of $9(28 \%)$ ranged between $3.5-4 \mathrm{~kg}$.

Clinical staging: In this study out of a total of 50 children, on the initial clinical staging,11 (22\%) had Stage I, $30(60 \%)$ had stage II and $9(18 \%)$ had stage III hypoxic-ischemic injury.

Clinical presentation: In this study of the total 50 children, the most common clinical presentation was respiratory distress seen in 35 (70\%) newborns followed by convulsions in 24 (48\%) and cyanosis in $13(26 \%)$ newborns.

Risk factors: Of the total newborns, the most common perinatal risk factor for asphyxia was found to be premature rupture of membranes in 7 (14\%) neonates followed by Antepartum hemorrhage in 6 $(12 \%)$. The other risk factors seen were Preeclampsic Toxemia in $5(10 \%)$, maternal hyper tension in $4(8 \%)$, oligohydramnios in $3(6 \%)$ and maternal diabetes mellitus in $2(4 \%)$ cases. No perinatal risk factor could be found in 23 (46\%) cases.
Table 1: Pattern of injury on TCUS

\begin{tabular}{|l|l|l|l|}
\hline & \multicolumn{1}{|c|}{ Overall } & Preterms & \multicolumn{1}{c|}{ Terms } \\
\hline White matter injury (WMI) & $14(28 \%)$ & $9(50 \%)$ & $5(15.62 \%)$ \\
\hline GMH(Germinal Matrix Hemorrhage) & $5(10 \%)$ & $5(27.77 \%)$ & $0(0 \%)$ \\
\hline Central & $9(18 \%)$ & $0(0 \%)$ & $9(28.12 \%)$ \\
\hline Mixed & $4(8 \%)$ & $0(0 \%)$ & $4(12.5 \%)$ \\
\hline Normal & $16(32 \%)$ & $3(16.66 \%)$ & $13(40.62 \%)$ \\
\hline Others & $2(4 \%)$ & $1(5.55 \%)$ & $1(3.12 \%)$ \\
\hline Total & $50(100 \%)$ & $18(100 \%)$ & $32(100 \%)$ \\
\hline
\end{tabular}

Comparison between TCUS findings and clinical staging of HIE: In our study among 9 (81.82\%) cases of HIE stage I, 17 (56.67\%) cases of HIE stage II and $8(88.89 \%)$ cases of HIE III had abnormalities on TCUS. These results were statistically significant.

Table 2: Pattern of injury on MRI

\begin{tabular}{|l|l|l|l|}
\hline & \multicolumn{1}{|c|}{ Overall } & \multicolumn{1}{c|}{ Preterms } & \multicolumn{1}{c|}{ Terms } \\
\hline WMI & $9(18 \%)$ & $8(44.44 \%)$ & $1(3.12 \%)$ \\
\hline GMH & $5(10 \%)$ & $5(27.77 \%)$ & $0(0 \%)$ \\
\hline Central & $15(30 \%)$ & $2(11.11 \%)$ & $13(40.62 \%)$ \\
\hline Mixed & $7(14 \%)$ & $1(5.55 \%)$ & $6(18.75 \%)$ \\
\hline Peripheral & $8(16 \%)$ & $0(0 \%)$ & $8(25 \%)$ \\
\hline Normal & 6 & $2(11.11 \%)$ & $4(12.5 \%)$ \\
\hline Total & $50(100 \%)$ & $18(100 \%)$ & $32(100 \%)$ \\
\hline
\end{tabular}

The spectrum of MRI findings: In this study, non-punctate white matter lesions were the most common finding on MRI observed in 14 (28\%) cases followed by intra/extra-axial haemorrhage in 13 (26\%) cases. Abnormal signal intensity in basal ganglia were observed in 12 (24\%) and thalamus in $11(22 \%)$ cases. The other findings observed were cortical highlighting, punctate white matter lesions were seen in $6(12 \%)$ cases each, restricted diffusion and border zone infarcts in $5(10 \%)$ and $3(6 \%)$ cases respectively.

Comparison between MRI findings and clinical staging of HIE: In our study among 9 (81.82\%) cases of HIE stage I, $26(86.67 \%)$ cases of HIE stage II and all cases of HIE stage III had abnormal MRI findings. These results were statistically significant.

Table 3: Comparison between TCUS and MRI findings as per the pattern of injury

\begin{tabular}{|c|c|c|c|c|c|c|c|c|c|}
\hline \multirow[t]{2}{*}{ TCUS } & \multicolumn{7}{|c|}{ MRI } & \multirow[t]{2}{*}{$x_{2}$} & \multirow[t]{2}{*}{ p-value } \\
\hline & Central & Peripheral & Mixed & GMH & WMI & Normal & Total & & \\
\hline Central & 9 & 0 & 0 & 0 & 0 & 0 & 9 & 0.00 & 1.00 \\
\hline WMI & 0 & 2 & 0 & 1 & 8 & 3 & 14 & 4.00 & 0.045 \\
\hline Mixed & 0 & 0 & 4 & 0 & 0 & 0 & 4 & 4.50 & 0.034 \\
\hline GMH & 0 & 0 & 1 & 4 & 0 & 0 & 5 & 6.00 & 0.014 \\
\hline
\end{tabular}




\begin{tabular}{|l|l|l|l|l|l|l|l|l|l|}
\hline Others & 0 & 1 & 0 & 0 & 1 & 0 & 2 & 1.00 & 0.317 \\
\hline Normal & 6 & 5 & 2 & 0 & 0 & 3 & 16 & 4.17 & 0.041 \\
\hline Total & 15 & 8 & 7 & 5 & 9 & 6 & 50 & & \\
\hline
\end{tabular}

In our study, the central pattern of injury was observed in 6 cases on TCUS further confirmed on MRI. Out of a total of 14 cases who were diagnosed with white matter injury on TCUS, MRI findings were consistent with white matter injury in 8 , peripheral pattern in 2, GMH in 1 case whereas 3 cases of these with increased periventricular echogenicity on TCUS were found to be normal on MRI. 4 children were diagnosed with a mixed pattern of injury on TCUS and further confirmed on MRI.
Of the total 5 cases with GMH diagnosed on TCUS, 4 cases had similar findings on MRI whereas 1 case showed additional findings and thus was diagnosed to have a mixed pattern of injury. 13 cases were falsely diagnosed normal on TCUS had a central pattern in 6 , peripheral pattern in 5 and mixed pattern in 2 cases on MRI. One case with aneurysmally dilated Vein of Galen on TCUS revealed additional bilateral parieto-occipitotemporal hemorrhages on MRI and another case with a right parieto-occipital haematoma on TCUS, MRI in addition diagnosed white matter hyperintensities and periventricular leukomalacia. Thus the results were statistically significant for mixed, GMH and white matter injury and non-significant for a central pattern of injury in HIE

\section{Table 4: Overall comparison between TCUS and MRI findings}

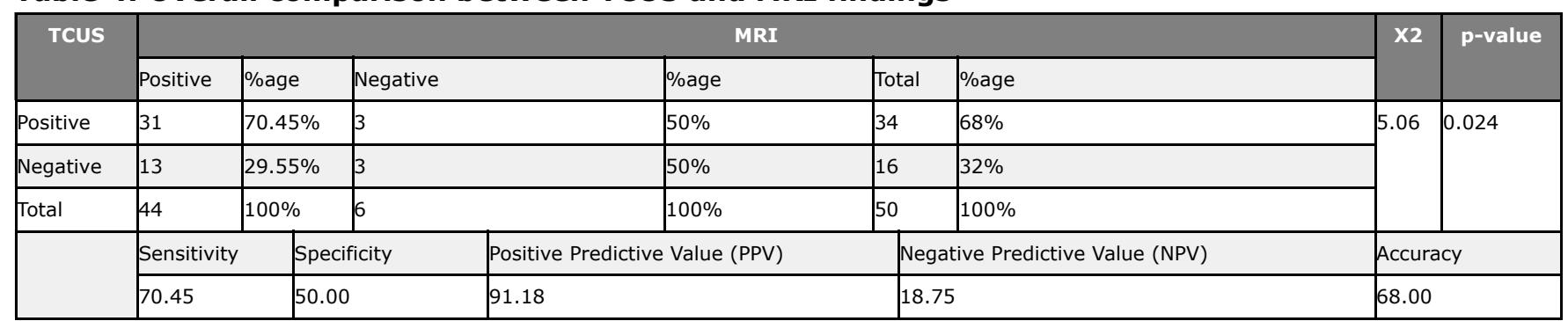

Thus there was a statistical difference detected between TCUS and MRI with higher efficacy of MRI in the diagnosis of neonatal HIE ( $p$-value $=0.024)$.

\section{Discussion}

In this study, white matter injury was found to be the predominant pattern of injury detected on TCUS followed by a central pattern of injury. A positive correlation was seen in the study by Bundi LB [6] in 2020 on 45 neonates in which periventricular and/or deep white matter echogenicity was the most common abnormality reported on cranial ultrasound followed by the thalamus and/or basal ganglia deep grey matter echogenic changes.

On MRI, the central pattern of injury was found to be the most predominant pattern seen followed by white matter injury. These findings were compatible with a study by Liauw L et al [7] in 2008 that showed that the most frequent early MRI finding in HIE neonates is BG (Basal Ganglia) brightness in T1-weighted images. In a study done by Genedi et al [8] in 2016 conducted on 38 neonates with HIE, 33 cases (86.9\%) showed abnormal findings; central pattern injury seen in $16(42.5 \%)$ and white matter injury noted in 4 cases $(10.5 \%)$.
One case $(2.6 \%)$ elicited a mixed pattern and the other two cases (5.2\%) elicited germinal matrix haemorrhage. Our results were in concordance with a study conducted by Aun AE [9] in 2019 in which out of 30 positive cases, the central pattern of injury was detected in $41.6 \%$, white matter injury seen in $18.3 \%$, peripheral injury in $27.8 \%$. One case $(2.8 \%)$ each elicited mixed pattern and GMH.

In preterms, white matter injury particularly periventricular leukomalacia was the most commonly detected abnormality on both TCUS and MRI while basal ganglia thalamus injury (central pattern) was the most commonly detected abnormality in term-born babies. A positive correlation was seen in a study conducted by Jose 0 [10] in 2017 in which $43 \%$ of preterm babies had periventricular leucomalacia. Also, MRI suggestive of haemorrhage was seen in preterm babies which constitute $7.4 \%$ of all babies with positive MRI. The study concluded that the preterm brain is highly susceptible to injury including periventricular leucomalacia, intraventricular hemorrhage/ germinal layer hemorrhage and parenchymal hemorrhagic infarction. $65 \%$ of term babies had changes in basal ganglia and/or thalamus. 
Term infants who develop HIE following a well defined acute hypoxic injury typically sustain bilateral lesions within the basal ganglia and thalami. Similarly study done by Bhagat $\mathrm{H}$ [11] in 2017 concluded that preterm infants have periventricular leukomalacia type of predominant pattern of involvement and term infants have significant involvement of basal ganglia thalamus type pattern in HIE.

In this study, the results were found to be statistically significant for mixed, GMH and white matter injury and non-significant for a central pattern of injury in HIE. TCUS could depict central and white matter abnormalities better than peripheral lesions in patients with HIE. In a study done by Genedi et al [8] in 2016 on 36 neonates with neonatal encephalopathy, TCUS accurately diagnosed all cases of germinal matrix haemorrhage. In correlation with MRI, TCUS had better sensitivity for detecting thalamic, basal ganglia and periventricular white matter lesions rather than lesions in the corpus callosum, brainstem, cerebral white matter, cerebral cortex and subcortical white matter. Our study findings were concordant with Steggerda et al [12] in 2009 who showed that TCUS has good sensitivity in detecting central and white matter injuries in neonates with HIE rather than peripheral injuries.

\begin{tabular}{|l|l|l|l|l|l|}
\hline & Sensitivity & Specificity & PPV & NPV & $\begin{array}{l}\text { Diagnostic } \\
\text { accuracy }\end{array}$ \\
\hline Present study & $70.45 \%$ & $50 \%$ & $\begin{array}{l}91.18 \\
\%\end{array}$ & $\begin{array}{l}18.75 \\
\%\end{array}$ & $68 \%$ \\
\hline $\begin{array}{l}\text { Genedi EA } \\
\text { (2016) [8] }\end{array}$ & $81.3 \%$ & $60 \%$ & $\begin{array}{l}93.1 \\
\%\end{array}$ & $\begin{array}{l}33.3 \\
\%\end{array}$ & $78.9 \%$ \\
\hline $\begin{array}{l}\text { Giri S. (2020) } \\
{[13]}\end{array}$ & $78.57 \%$ & $62.5 \%$ & $76 \%$ & $\begin{array}{l}35.7 \\
\%\end{array}$ & $76 \%$ \\
\hline
\end{tabular}

Table 5: Transcranial ultrasound as compared to MRI

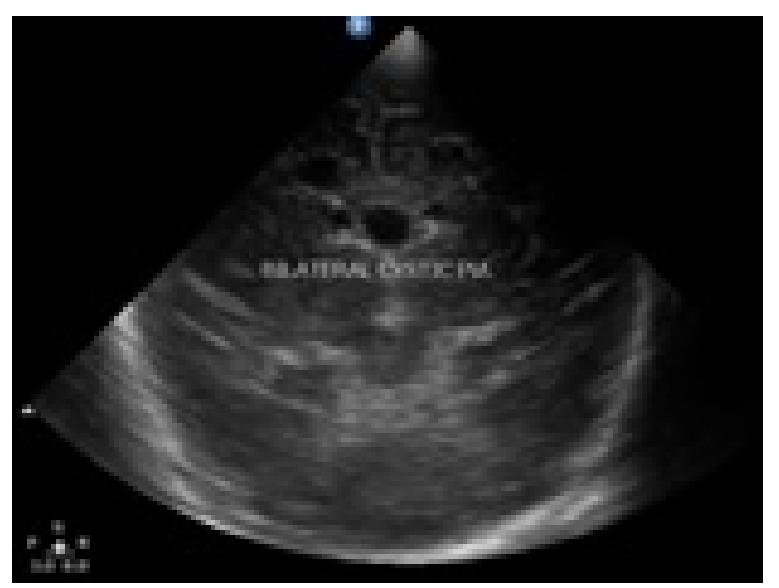

Figure 1(A)

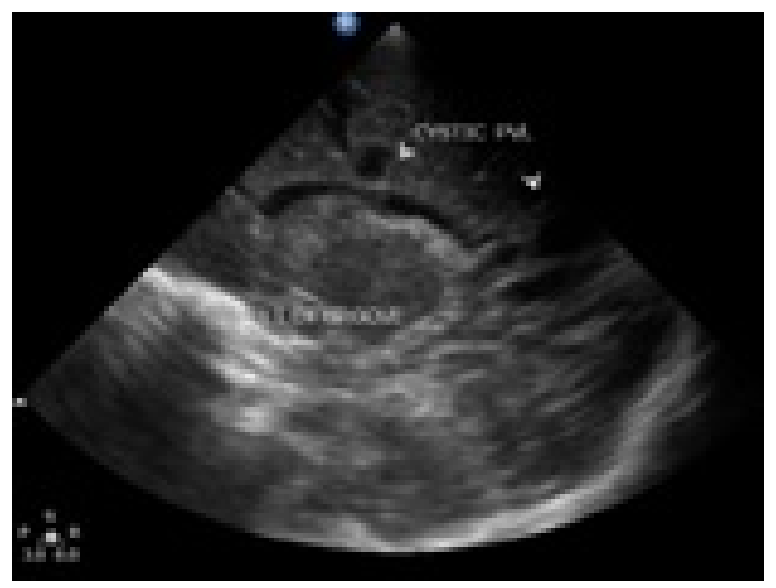

Figure 1(B)

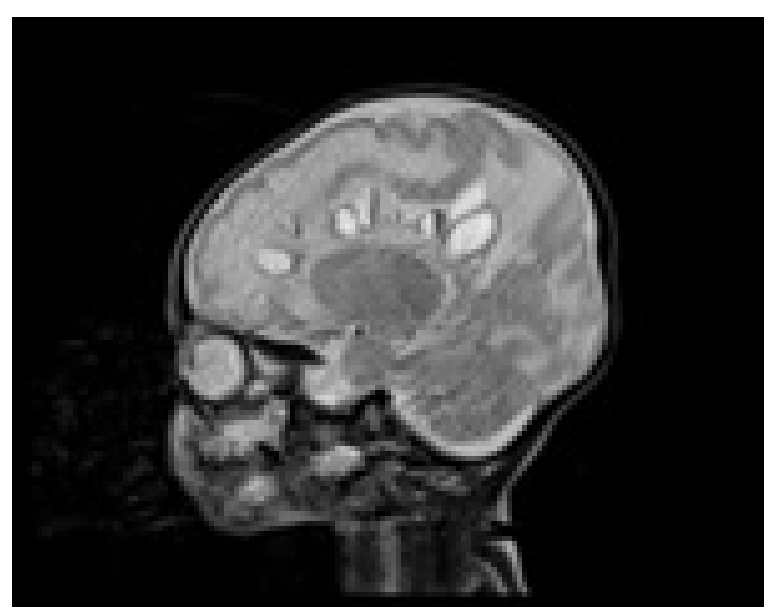

Figure 1(C)

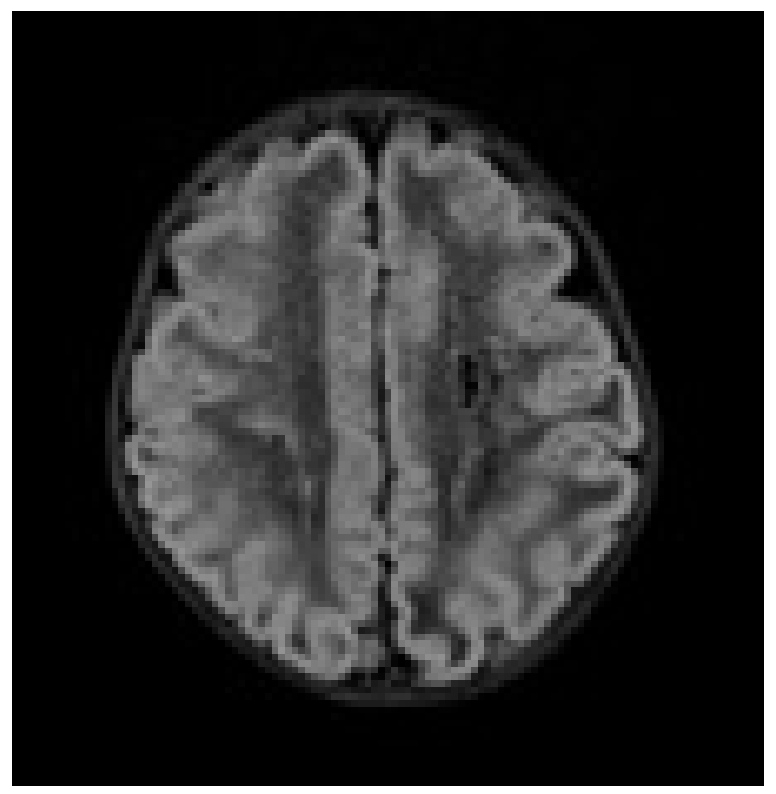

Figure 1(D) 
Figure 1: 28 day male neonate born as preterm by NVD with perinatal asphyxia.

(A): Coronal and

(B): Left parasagittal transcranial ultrasound images showing bilateral periventricular cysts around frontal horns of lateral ventricles.

(C): T2 sagittal and

(D): T2 FLAIR axial MRI images depicting bilateral cystic periventricular leukomalacia.

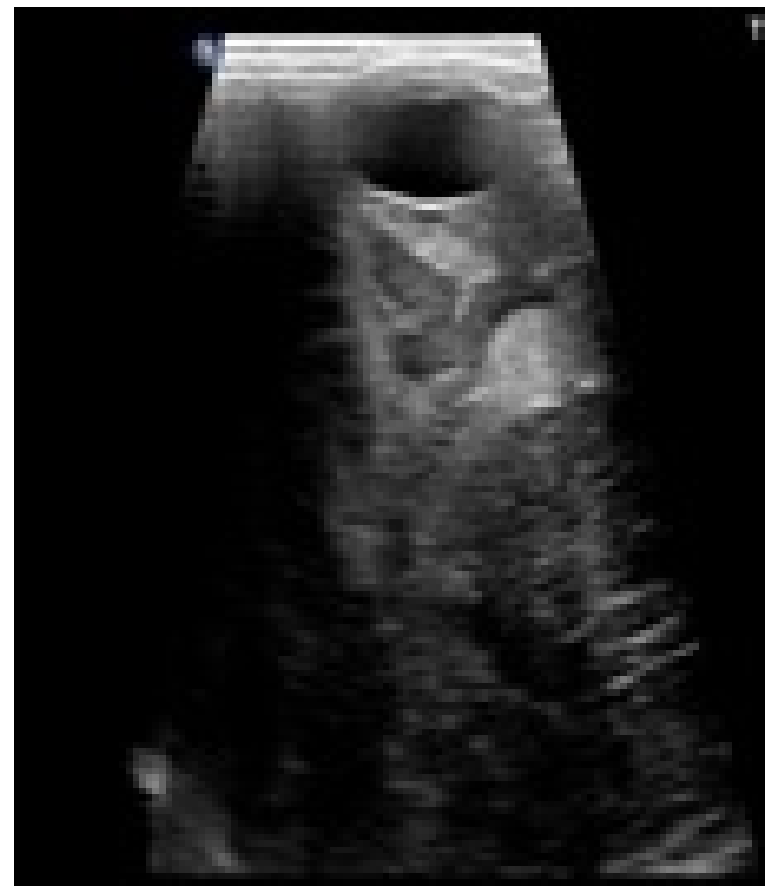

Figure 2(A)

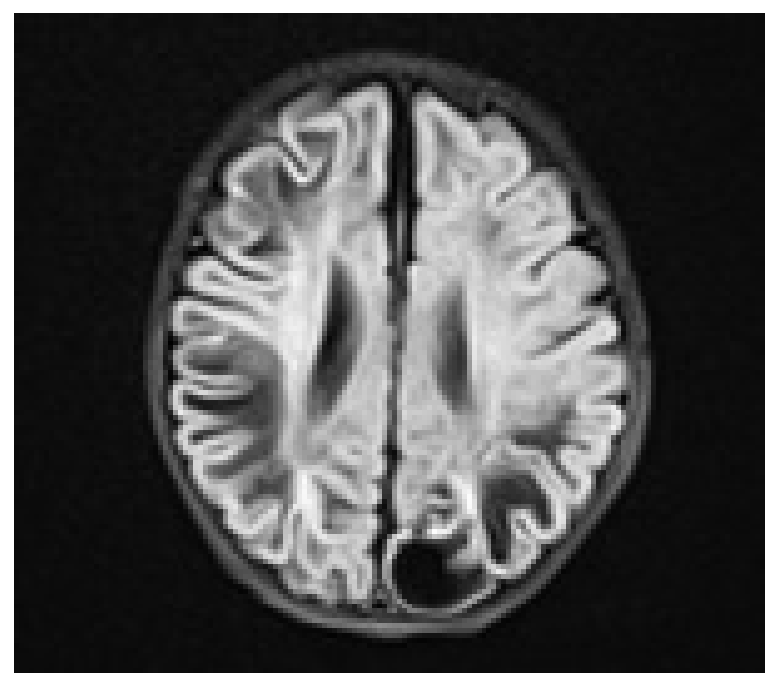

Figure 2(B)

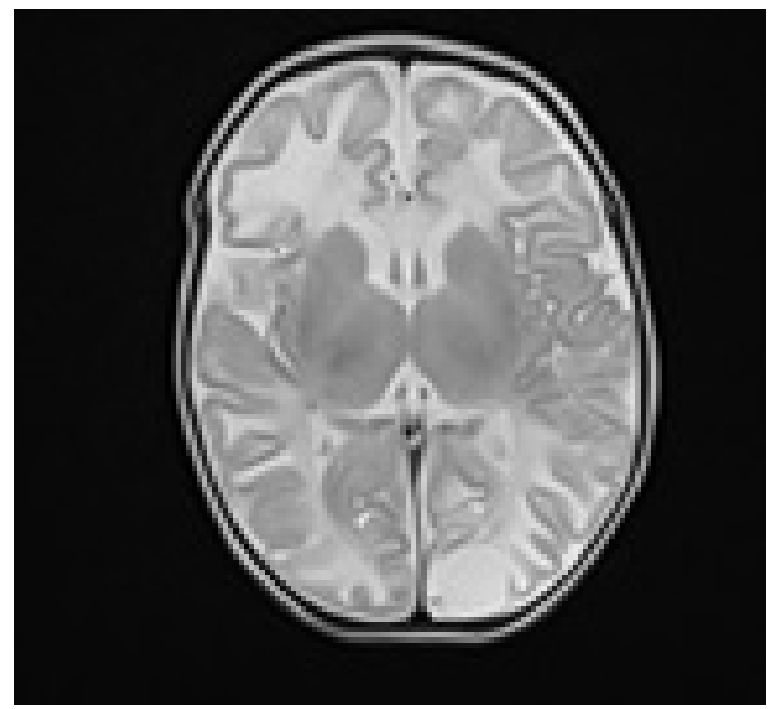

Figure 2(C)

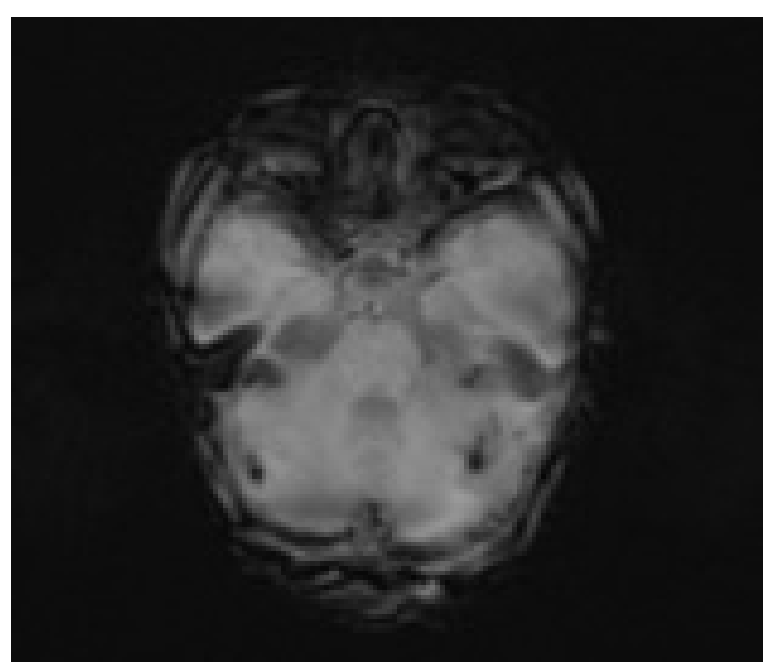

Figure 2(D)

Figure 2: 1-month 2-day female baby born as a term by LSCS with apneic spells and seizures at birth.

(A): Coronal transcranial ultrasound through posterior fontanel depicting thin-walled cysts in leftbrain parenchyma.

(B) : T2 FLAIR axial and

(C): T2 axial MRI images showing extensive areas of cystic encephalomalacia involving bilateral cerebral hemispheres.

(D): Axial SWI image showing foci of blooming in bilateral cerebellar hemispheres indicative of haemorrhagic foci. 


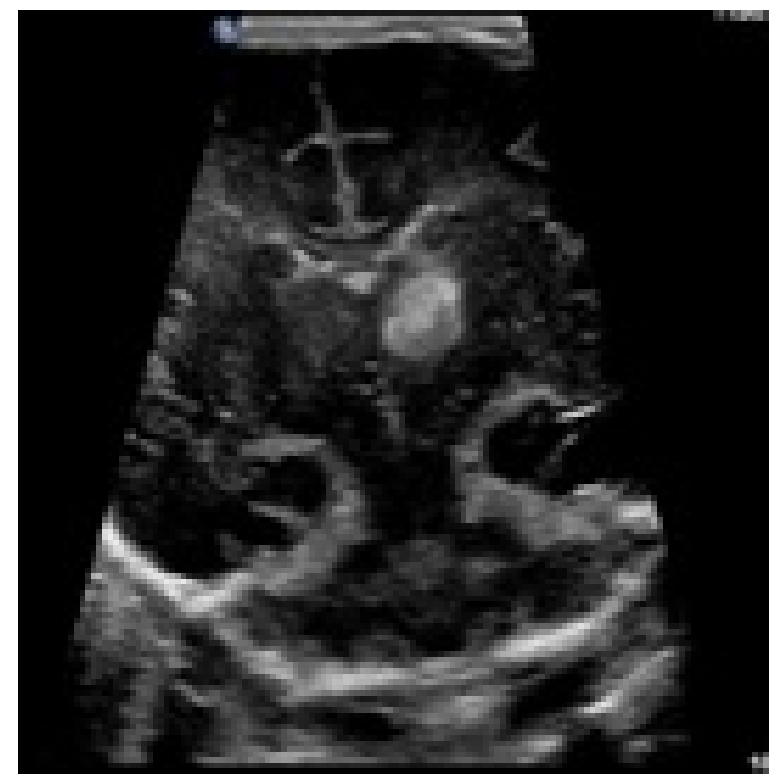

Figure 3(A)

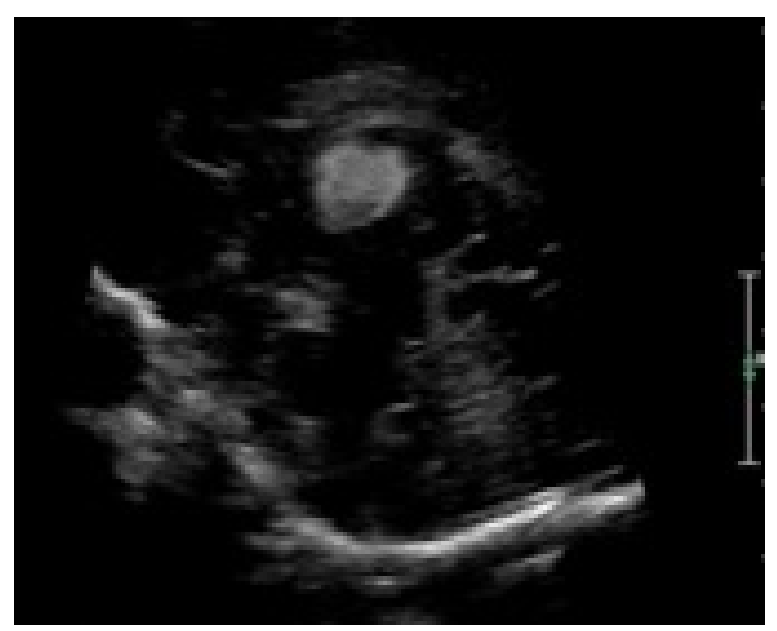

Figure 3(B)

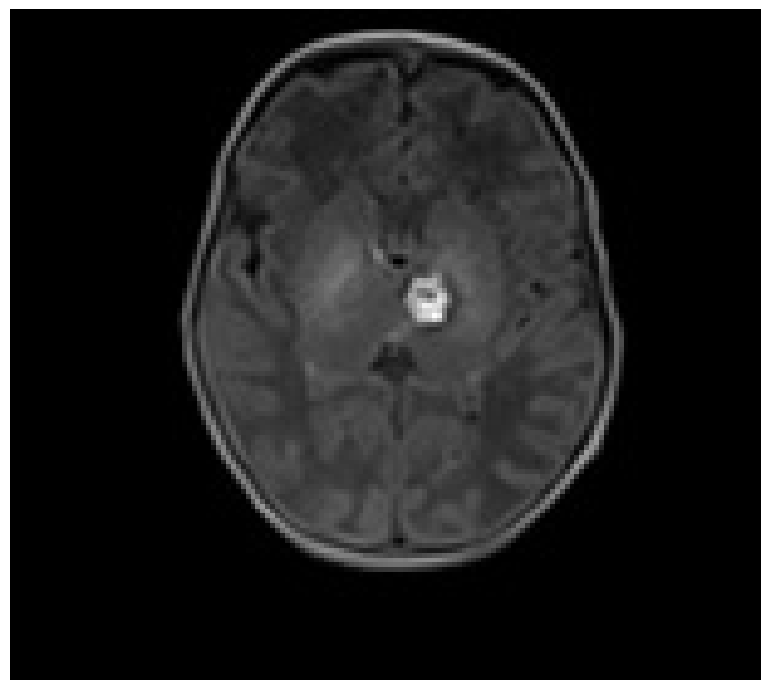

Figure 3(C)

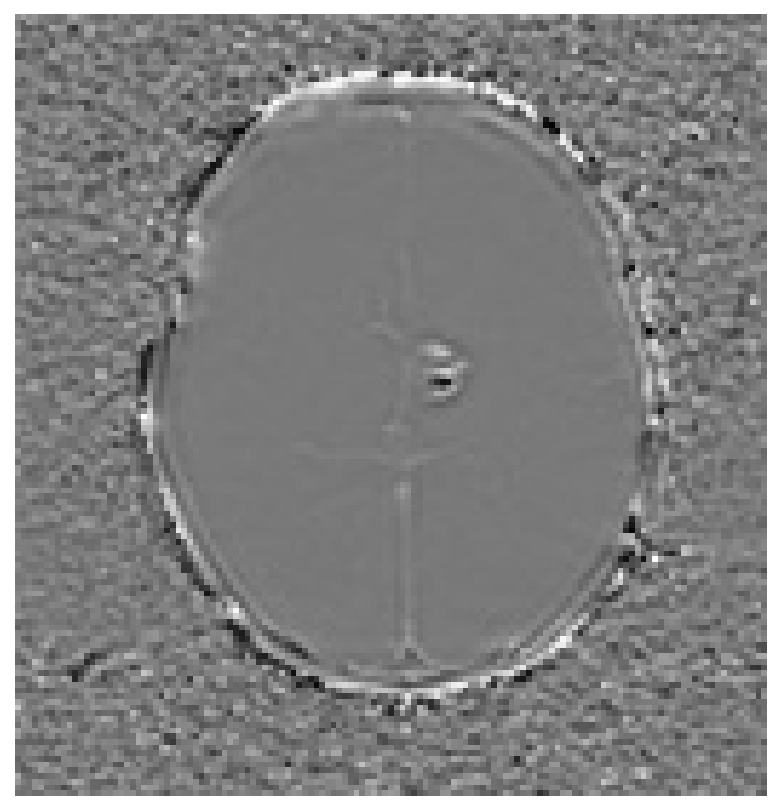

Figure 3(D)

Figure 3: 13-day male baby delivered as preterm by NVD with respiratory distress at birth. (A): Coronal and (B): Left parasagittal transcranial ultrasound showing an echogenic area in left caudothalamic groove consistent with germinal matrix haemorrhage. (C) : T1 axial image showing hyperintense focus in the left caudothalamic groove which blooms on corresponding SWI image (D) indicating grade I GMH.

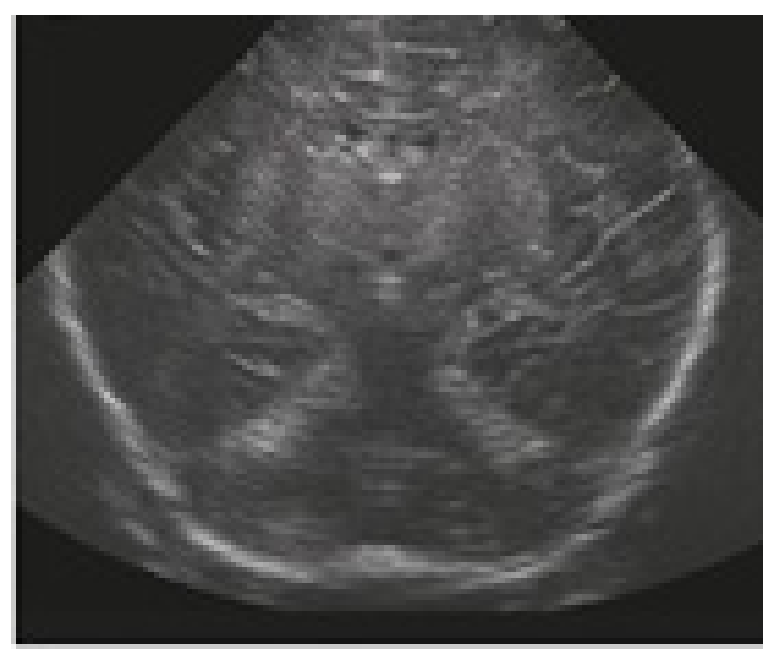

Figure 4(A) 


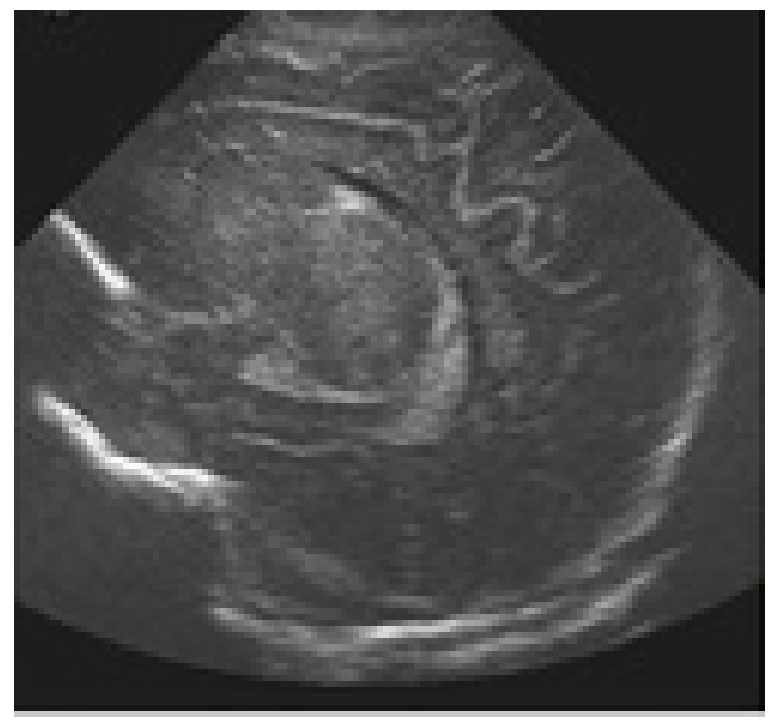

Figure 4(B)

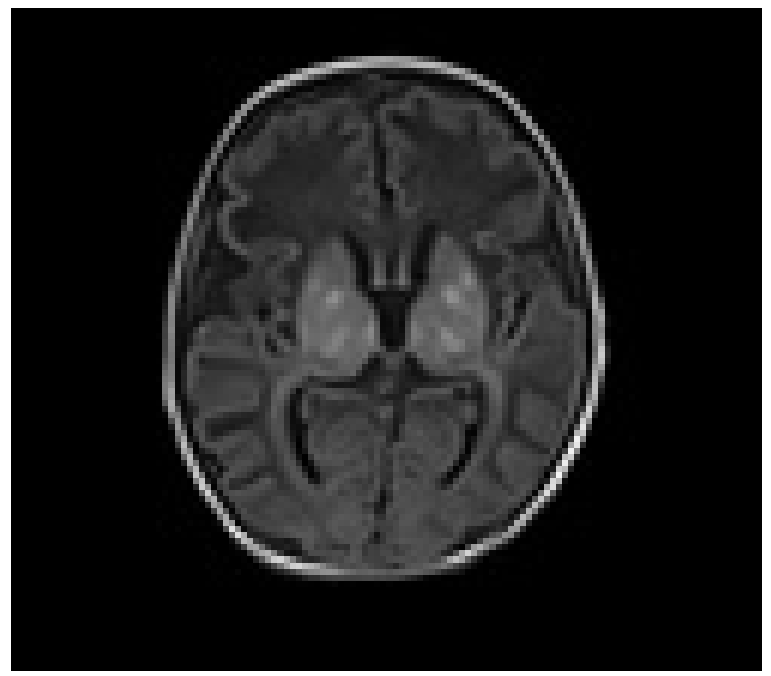

Figure 4(C)

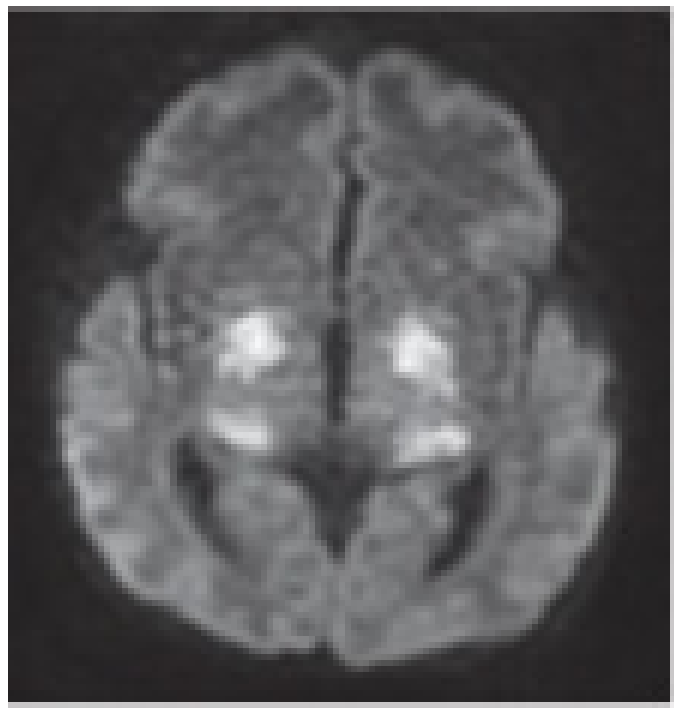

\section{Figure 4(D)}

Figure 4: 21-day male born at term presented with respiratory distress, apneic spells and seizures at birth. (A): Coronal and (B): Parasagittal transcranial ultrasound images showing increased echogenicity of bilateral basal ganglia and thalami. (C): Axial T1WI showing multiple foci of hyperintensity in bilateral basal ganglia and thalami. (D): Axial DWI image showing the area of restricted diffusion in bilateral basal ganglia and thalami. These findings were consistent with basal ganglia thalamic type of injury.

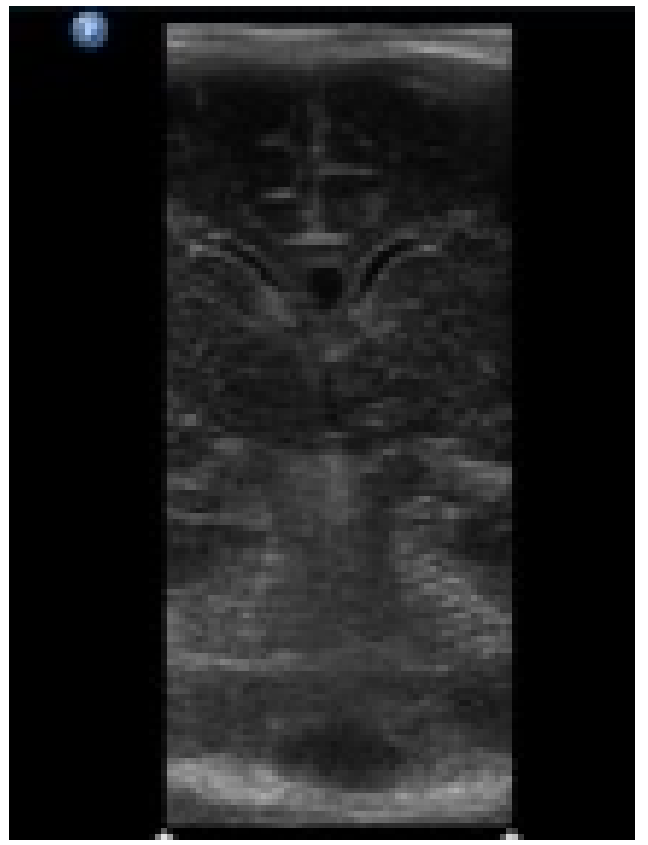

Figure 5(A)

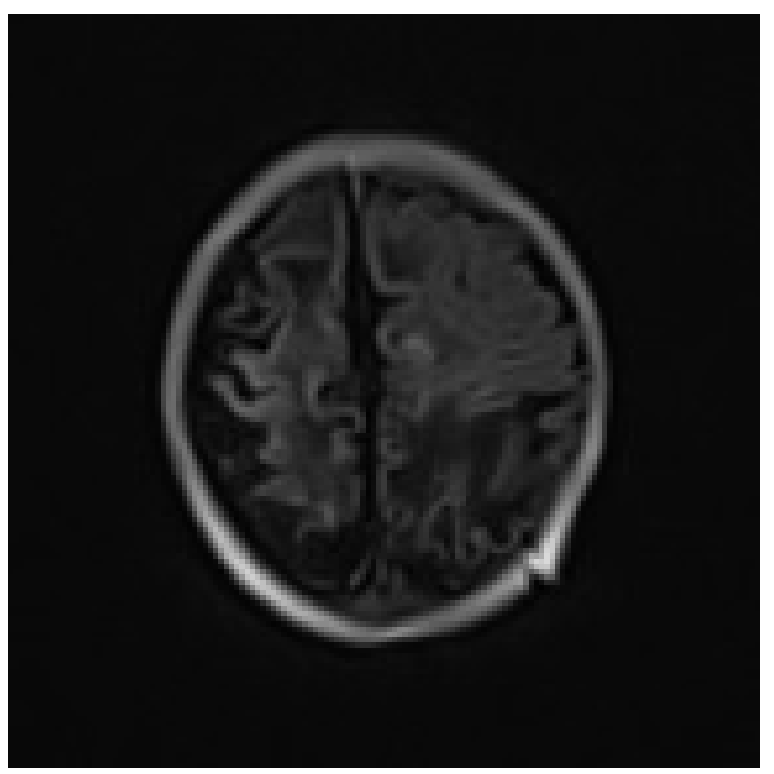

Figure 5(B) 


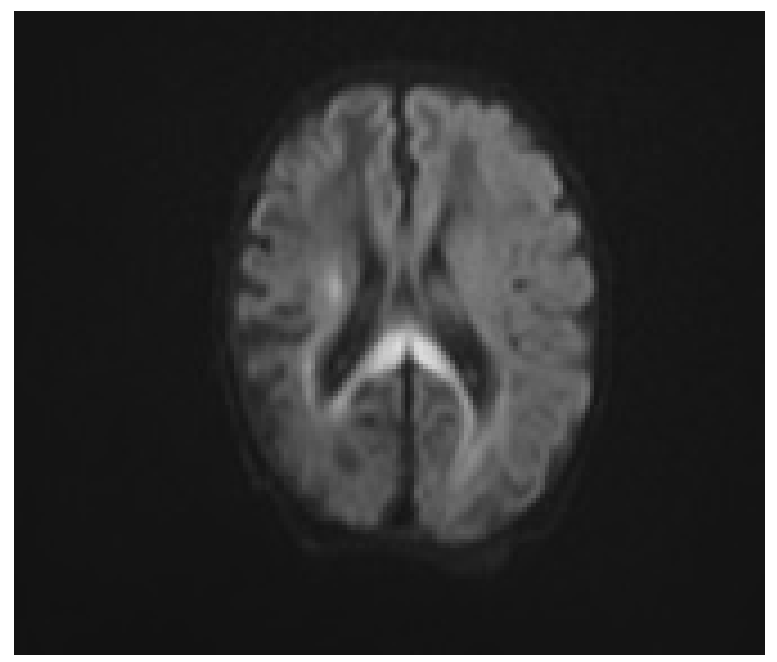

Figure 5(C)

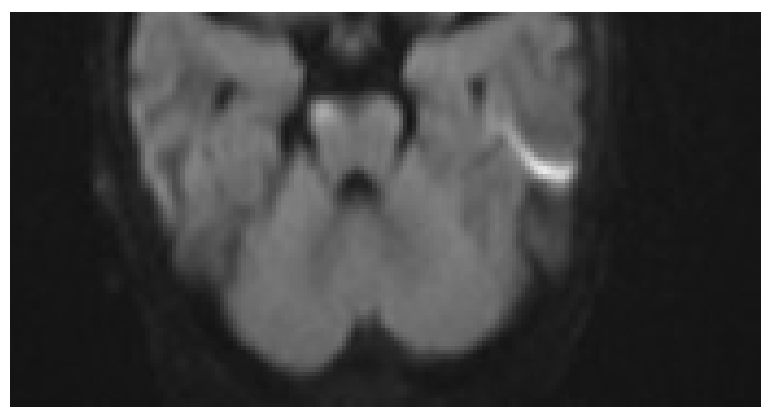

Figure 5(D)

Figure 5: 28 days female born at term by NVD with severe birth asphyxia, apneic spells and early-onset neonatal sepsis.

(A): Coronal transfontanel ultrasound showed the grossly normal study.

(B): Axial T1 blade image showing gyriform curvilinear hyperintensities in bilateral cerebral hemispheres s/o cortical laminar necrosis.

(C) and

(D): Axial DWI images showing restricted diffusion in the posterior limb of the right internal capsule, splenium of the corpus callosum and right corticospinal tracts - likely representing Pre Wallerian degeneration.

\section{Conclusion}

The imaging spectrum of HIE is wide depending upon the time and severity of hypoxic insult, ranging from periventricular leukomalacia, germinal matrix hemorrhage to deep grey matter infarction in preterms and parasagittal cortical-subcortical injury to basal ganglia thalami injury in term neonates.
This study demonstrated term infants have significant involvement of basal ganglia thalamus type pattern of involvement and preterm infants have periventricular leukomalacia type of pattern of involvement. Both transcranial ultrasound and MRI are useful imaging modalities for neonates with hypoxic-ischemic injury. TCUS can depict central and white matter abnormalities better than peripheral lesions. MRI provides additional diagnostic information in many cases and can detect precisely the extent of brain injury. DWI often reveals ischemic injury at an earlier stage compared to conventional MRI.

\section{Limitations of the study}

The only limitation of this study was a small sample size. A larger population cohort is desirable to achieve more accurate results.

\section{Author contributions}

Dr. Maonj Mathur, Dr. Ishita Gupta, Dr. Dimple Mittal all contributed equally in the conduct of the study and the preparation of the manuscript.

\section{Reference}

01. Thoker AH, Sheikh M, Thoker PA, Thoker M. Neurodevelopmental outcome in perinatal asphyxia. American Journal of Experimental and Clinical Research. 2017 Apr 8;4(2)206-9.

[Crossref]

02. Pathiraja RP, Guneskera D. Perinatal asphyxia and Hypoxic Ischemic Encephalopathy-The Current Situation. Sri Lanka Journal of Obstetrics and Gynaecology. 2017;39(1)8-11. [Crossref]

03. Vélez SM, García JM, Marín LC, Arango JM, White CA, Pineda DS. Hypoxic ischemic encephalopathy in the neonatal period, evaluation with magnetic resonance. Rev Colomb Radiol. 2018;29(4)5025-31.

[Crossref]

04. Pathiraja RP, Guneskera D. Perinatal asphyxia and Hypoxic Ischemic Encephalopathy-The Current Situation. Sri Lanka Journal of Obstetrics and Gynaecology. 2017;39(1)8-11. [Crossref]

05. Rutherford MA. The asphyxiated term infantMRI of the neonatal brain. London- WB Saunders. 2002 Jan;99-128.

[Crossref] 
06. Bundi LB, Mwango G, Oliver VO, Mulama B. Clinical neonatal hypoxic ischemic injuryCranial ultrasound spectrum of findings in neonates admitted to a Newborn Unit in Nairobi, Kenya. West African Journal of Radiology. 2020 Jul $1 ; 27(2) 108$.

[Crossref]

07. Liauw L, van der Grond J, van den BergHuysmans AA, Palm-Meinders $\mathrm{IH}$, van Buchem $M A$, van Wezel-Meijler G. Hypoxic-ischemic encephalopathy- diagnostic value of conventional MR imaging pulse sequences in term-born neonates. Radiology. 2008 Apr;247(1)204-12.

doi: 10.1148/radiol.2471070812 [Crossref]

08. Genedi EA, Osman NM, El-deeb MT. Magnetic resonance imaging versus transcranial ultrasound in early identification of cerebral injuries in neonatal encephalopathy. The Egyptian Journal of Radiology and Nuclear Medicine. 2016 Mar 1;47(1)297-304.

[Crossref]

09. Aun AE, Hassan HA, Ali WI, Ataky MM. Transcranial Ultrasound in Comparison to MRI in Evaluation of Hypoxic Ischemic Injury in Neonates. The Egyptian Journal of Hospital Medicine. 2019 Jan 1;74(4)842-52.

[Crossref]
10. Jose O, Sheena V. MRI changes of brain in newborns with hypoxic ischemic encephalopathy clinical stage II or stage III-a descriptive study. International Journal of Medical Paediatrics and Oncology. 2017;3(1)29-33.

[Crossref]

11. Bhagat $H$, Kawade R, Sachdev YP. Study of role of MRI brain in evaluation of hypoxic ischemic encephalopathy. Indian Journal of Basic and Applied Medical Research, Radiology and Imaging. Dec 2017;7(1)28-33.

[Crossref]

12. Steggerda SJ, Leijser LM, Wiggers-de Bruïne FT, van der Grond J, Walther FJ, van Wezel-Meijler G. Cerebellar injury in preterm infants: incidence and findings on US and MR images. Radiology. 2009 Jul;252(1)190-9. doi: 10.1148/radiol.2521081525 [Crossref]

13. Giri S, Sau R, Das S, et I. Correlation of transcraial ultrasound and magnetic resonance imaging in evaluation of imaging patterns of clinically diagnosed hypoxic ischemic encephalopathy in neonates. J Evid Based Med Healthc. 2020;7(19)938-42.

[Crossref] 